\title{
SOME FACTS BEARING ON THE STRUCTURE OF ATOMS, PARTICULARLY OF THE HELIUM ATOM.
}

\author{
By R. A. Millikan.
}

SyNOPSIS.

\begin{abstract}
Discussion of Suggested Models for Helium and Other Atoms in the light of recent results of a study of multiple ionization by alpha rays. As will be reported more fully elsewhere it has been found that slow alpha rays detach both electrons from some of the helium atoms through which they pass, the number of doubles being one sixth the number of singles, while in the case of the other gases tested no evidence of multiple ionization has been obtained. The result is considered by the author to indicate that the electronic orbits in the helium atom must be such as to bring the two electrons into the same part of the atomic volume at least one sixth of the time, a condition which is well satisfied by the recent models of Landé and Bohr in which the orbits cross each other. In the case of other atoms the new evidence favors the Bohr models, with few electrons in the outer shells.

Multiple Ionization in Arcs and Sparks is Probably Due to successive ionization
\end{abstract} of the same atoms.

I ET $p$, Fig. I, be a minute oil drop, carrying for example one free positive electron and let the strength of a powerful electrical field between the plates $A$ and $B$ be adjusted until the weight of the drop is exactly balanced by the pull upon it of the electrical field. Then by opening the shutter $S$ let an alpha particle from a bit of polonium at $a$,

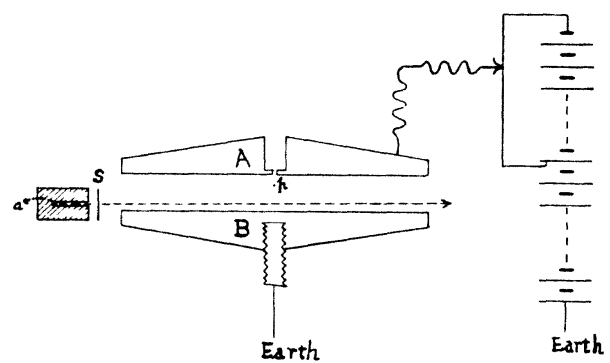

Fig. 1. Fig. I, be shot underneath the drop. The atoms of the gas underneath $p$ have electrons knocked out of them by the passage of the alpha rays through them and the positively charged residue of such an atom as happens to be immediately underneath the drop is thrown instantly to the latter, where the charge carried by that residue is at once determined by the speed with which the drop begins to move upward. In the actual experiments which have been carried out by this method at the Ryerson Laboratory, ${ }^{1}$ the catching of an atom from which one electron had been removed by an alpha or beta particle

\footnotetext{
1 Phil. Mag. (6), 21, 753, r9I1. Phys. Rev., I5, I57, r920.
} 
has in general caused the drop to move with a speed of I scale division in about 14 seconds. If the residue of the atom caught by the drop has had 2 electrons removed from it, the drop moves over one division in seven seconds, 3 electrons causes it to move over one division in 4.7 seconds, etc.

By observing, then, the speed corresponding to each catch, it is possible to determine without the slightest ambiguity exactly how many electrons have been removed from an atom by the passage of an alpha particle through it. As soon as a catch is observed, the shutter $S$ is thrown across the path of the alpha rays so as to prevent further ionization until the measurements upon the last catch have been completed.

Results by this method have already been published ${ }^{1}$ which reveal the interesting fact that a swift moving alpha particle practically never succeeds in detaching more than one electron from a given atom through which it passes whether that atom is one of the lighter ones like carbon, nitrogen, or oxygen, which possess but from 6 to 8 electrons, or whether it is one of the heavier ones like iodine or mercury, the latter of which possesses as many as 82 electrons. This, in itself, throws a certain amount of light on atomic structure, for it shows conclusively that the electrons within an atom act quite independently of one another. They are certainly not in rings, of say 4 or 8 or any other number which become unstable when one of their number is removed, or which can in general be shattered as a whole.

In view, however, of the fact that the ionizing power of an alpha particle is an inverse function of its speed, and also of the fact that J. J. Thomson's positive ray photographs show unambiguous evidence of multiple-ionization of certain types of atoms, I have recently started, as promised in a former paper, ${ }^{2}$ some new experiments of the type described above save that the range of the alpha particle within the chamber was so adjusted, by introducing sheets of aluminium foil into its path, as well as varying amounts of gas, that it could be brought underneath the drop at the very end of its range where its ionizing power reaches as high a value as it can ever attain. These observations have been carried out in the Ryerson Laboratory chiefly in collaboration with Mr. T. R. Wilkins, who has carried the number of catches up into the thousands, but I have myself made sufficient observations to obtain an independent check upon the correctness of the main results obtained. The details of the observations will be reported by him in a following article, but the results may be summarized and discussed as follows:

I. In the case of helium, the alpha particle, when it is in that part of its range which corresponds to its maximum ionizing power, detaches

1 Phys. Rev., I5, I57, 1920.

${ }^{2}$ Phys. Rev., I5, I77, 1920. 
both of the two electrons that the atom of helium contains 16 per cent. ${ }^{1}$ of the time that it detaches either of them. Otherwise stated, the alpha particle shooting at random through the helium atom at its maximum ionizing speed, gets both electrons every sixth shot in which it gets anything.

2. The percentage of doubles produced in helium by the alpha particle increases from about 8 per cent. at the middle of the range up to a maximum of 16 per cent. which is reached at exactly the portion of the range which corresponds to the maximum ionizing power. In other words, the curve of doubles plotted against range follows, just as it ought to do, the curve of ionization plotted against range.

3. No atom thus far tested except that of helium shows any distinct evidence of multiple ionization by a single alpha particle, even when that particle is moving at the speed corresponding to its maximum ionizing power. The other atoms thus far tested, all in molecular combination, are those of oxygen, nitrogen, carbon, hydrogen and mercury.

Although the foregoing facts are not sufficient to determine in any way the structure of any atom, they are amply sufficient to render impossible certain sorts of suggested structures for the helium atom.

Thus, the original Bohr theory which placed the two electrons of the helium atom at the poles of a diameter, cannot be reconciled I think with these observations; for, since both electrons go out independently, otherwise we should not get one of them alone five times out of six, the alpha particle can only rob them both from the parent nucleus (which is also an alpha particle) if, in going through the atom, it comes closer to both electrons than the normal distance between each of them and the nucleus. Now, the speed with which the alpha particle is moving when it produces its maximum ionization comes out very nearly the same as the speed of rotation of the electrons within the helium atom, as computed with the aid of the Bohr theory. If, therefore, the alpha particle came close enough to one electron to catch it at one pole of a diameter, it would have very little chance, even when moving in the plane of the orbit, of catching the other electron at the other pole, since by the time it got across the orbit, the second electron would be about an orbital diameter away from it. If the alpha particle is moving at right angles to the plane of the orbit, it has no chance at all of catching both electrons. From whatever point of view this structure is approached, I cannot see that it permits both electrons to be detached by a single alpha particle one-sixth as frequently as either one of them is detached.

${ }^{1}$ The observations themselves give about 15 per cent., but the helium was but about 96 per cent. pure, and since the impurity was inert in this particular, it is computed that pure helium would show about 16 per cent. of doubles. 
Secondly, the facts herewith presented are also irreconcilable with a structure at one time suggested by Sommerfeld in which one electron rotates in an orbit close to the nucleus, while the other is in an orbit of very much greater diameter, for here again the chance that the alpha particle can come close enough to an electron to pull it away should vary at least as rapidly as the radius of the orbit, which would mean that the inner electron could not be detached one sixth as frequently as the outer unless its orbit were more than one sixth the diameter of the outer's orbit. The chance, then, that with any such ratio of orbits both electrons would be detached at once, would be exceedingly minute.

It is to be noted too that since the chance of an impact with the nucleus itself in such a way as to cause it to be jerked away from both its electrons is not a hundred-millionth $\left(\mathrm{IO}^{-12} / \mathrm{IO}^{-8}\right)^{2}$ of the chance of a passage of the alpha particle through the outer regions of the atom it is at once obvious that this way of accounting for doubles is altogether excluded.

In a word, then, the fact that every sixth shot which detaches any electron at all from the atom, gets them both, demands that both electrons find themselves in the same part of the atomic volume a very appreciable fraction of the time. This condition is well satisfied by the recent theories of Landé and of Bohr, according to which the two electrons are in orbits of much the same diameter but inclined to each other at angles of $60^{\circ}$ or $90^{\circ}$.

It is at first sight a matter of no little surprise that the helium atom, with but two electrons to lose, loses them both one-sixth of the time while the atoms with many electrons, in the case of mercury, 82, apparently have in general but one detached at a time by the passage of an alpha particle through them. This fact is of some significance for atomic structure. The evidence of the $\mathrm{K}$ series of $\mathrm{x}$-rays is to the effect that the structure of the helium atom repeats itself in the $\mathrm{K}$ ring or shell of all atoms. That the two electrons in this ring are not detached by alpha particles except in the case of helium is not surprising, since the ring shrinks rapidly in diameter as the atomic number increases, and since the forces holding these electrons to the nucleus also increase proportionally to the atomic number. That the larger number of electrons in the outer shells appear in practically all cases to be detached by alpha particles one at a time, indicates, first, a different structure in these shells from that existing in the inmost one, i. e., existing in the helium atoma difference quite in accord with the multiplicity of orbits indicated by the theories of Bohr and Sommerfeld. In the second place, it shows, quite independently of the theory of Bohr, but also altogether in accord with it, that in an atom like mercury the great majority of its 82 electrons 
are in intense fields of force close to the nucleus where the chance of the alpha particle coming close enough to them to rob them from that highly charged nucleus is exceedingly remote. It will be remembered that the elementary form of the Bohr theory requires that the radii of the $\mathrm{K}, \mathrm{L}$, $\mathrm{M}$, etc., rings or shells bear the ratios $\mathrm{I}, 4,9$, etc., and that these radii all shrink in the inverse ratio of the atomic number, while the observed frequencies in the $\mathrm{K}, \mathrm{L}, \mathrm{M}$ series indicate actual ratios of radii, computed from Bohr's equations, which are somewhat larger than the foregoing values.

The present group of experiments, then, constitute new evidence in favor of an atom of the general type postulated by Bohr in which the chance that an alpha particle could detach electrons from any save the outer shell is very remote. That it does not detach two at a time, even from the outer shells of the $\mathrm{N}, \mathrm{O}$, or $\mathrm{Hg}$ atoms, while it does frequently get them both in the case of helium, is perhaps to be expected from the larger diameters, and the more powerful nuclei of $\mathrm{N}, \mathrm{O}$, and $\mathrm{Hg}$ in comparison with $\mathrm{He}$, and from the imperfect shielding of these outer shells by the electrons of the inner shells. Bohr may also find in it support for his recent contention ${ }^{1}$ that the outer shell of heavy atoms possesses few electrons instead of many as postulated in most of the discussions of the "Static Atom."

In view of the failure to obtain multiple ionization in these experiments with oxygen, nitrogen, mercury and other of the heavier atoms, even when the alpha particles are moving with the slowest speeds, it appears not improbable that the multiple ionization observed by J. J. Thomson and others in connection with positive rays, and also the double ionization quite conclusively indicated by the differences between arc and spark spectra, may be due to the successive ionizations of the same atom by electrons or by radiations from adjoining atoms, or by both. These multiple ionizations are revealed where very dense electron currents exist, but with such dense electron currents there is no doubt about the existence of such successive ionizations both by the absorption of radiation and by multiple electronic impacts. Such an assumption is required for the explanation of the phenomena of low voltage arcs. Further, the higher the atomic number, the more chance there is for such multiple ionization, and it is to be noticed that a study of the multiple ionization obtained with positive rays, shows that the number of multiples increases with atomic number much as it should if that hypothesis is correct.

\footnotetext{
Norman Bridge Laboratory of Physics,

Pasadena, California.

${ }^{1}$ Nature, March 24, p. I04.
} 